\title{
A Study on Parthenogenesis of Petersen Graph
}

\author{
Siti Amiroch and Danang Kiratama
}

\begin{abstract}
Genetics is the science of trait from the parent to the descendant. In biology, genetics pass a series of genes unification process that takes place in the chromosome. The results of genes unification will form the nature and character of the generation. This particular genetic process also applies in graph theory. Genetics on graph theory is divided into two: breeding and parthenogenesis. This present study elaborated a single type of genetic processes that was parthenogenesis which is applied on a Petersen graph. Through the similar process to genetics in biology, Petersen graph will be reconstructed and combined with other graphs (gene) in purposes to create a descendant or a new graph with new nature and characteristic. Based on the result of parthenogenesis on this Petersen graph, there was derived a graph which has 18 edges and 12 vertices, isomorphism toward another Petersen graph, Hamiltonian, and has 3 girth and symmetric.
\end{abstract}

Index Terms-Genetics graph, parthenogenesis, Petersen graph.

\section{INTRODUCTION}

G RAPH theory is a study in mathematical science that discusses about discrete objects and the connectivity between those objects. The representation of the objects in Graph Theory is in form of dots (vertex) which are linked with line or side called edge [1].

A graph $(\mathrm{G})$ is defined as the pair set point $(V)$ and side $(E)$ that was written with the notation $G=(V, E)$, which in this case $(V)$ is not empty sets of vertices and $(E)$ is the side set connecting a pair of vertices [2]. Graf has many types which is one of them is a Cubic graph $(\Gamma)$. Cubic Graph $(\Gamma)$ is a graph with 3 sides in each vertex that connects between vertices. Besides of its many types, the graph also has many characteristics. One of its characteristics is symmetric. Graph can be said symmetric if Automorphism is congruent or applicable on that graph [3].

In biology, one of interesting materials to be studied is Genetics. A branch of biology that studies trait from parents (parental) to their generation and also a breeding of living creature is called genetics. In general, the breeding of the living creatures is classified into two which are sexually breeding and asexually breeding. Parthenogenesis is an asexually breeding process in which the breeding and the growing of the embryo occurs without insemination [4].

In addition to the biology, genetics also available in graph theory. Principles and rules of genetics have been investigated by [1]. Then, [5] elaborated genetic processes in Cubic Graf published in Genetic Theory for Cubic Graphs journal. In his

Manuscript received February 8, 2017; accepted February 17, 2017.

The authors are with the Department of Mathematics, Faculty of Mathematics and Natural Science, Universitas Islam Darul 'Ulum, Lamongan 62253, Indonesia. Email: amirast_117@yahoo.com, kierotomo@gmail. com journal, [5] stated that genetic processes in Cubic Graph are classified into two: the breeding and parthenogenesis.

In this study, by using this process of parthenogenesis, we would like to examine how the influence of parthenogenesis process applied to Petersen Graph. It is expected that this study can be a pioneer on the appearance of other studies on Graph theory in general and Genetics Graph in particular.

\section{Methods}

\section{A. Graph}

The definition of graph is a representation of set in which some or all of the objects associated are linked with link. The objects that are connected are called as point (vertex) and the link that connects the objects is called as side (edge) [6].

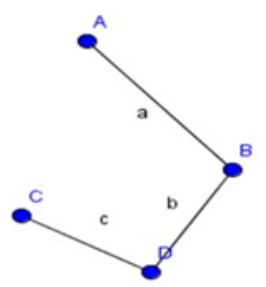

Fig. 1. Example of a graph.

\section{B. Graph Terminology}

Graph terminology is the nature and the connectivity between vertex and edge in a graph. In the connectivity between vertex and edge in the graph, two terms are known: adjacency (adjacent) and incidence (incidency). A vertex is said to neighborhood if the vertex is directly connected to the other vertices. Then, an edge is said to be incident when the edge is connected directly to the other edges.

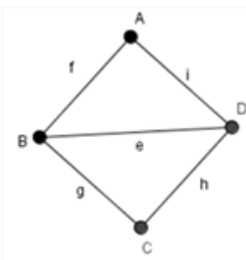

Fig. 2. Vertex $A$ is adjacent with vertex $B$ and vertex $D$, Edge $f$ is incident with edge $g$ and $i$.

\section{Cubic Graph}

Cubic Graph is a graph in which each vertex has degree three or it can be said that of each vertices in the graph is passed by three edges and is connected to all vertices [7] The degree of the graph is denoted by deg $(v)$ 


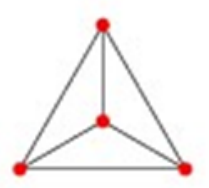

Fig. 3. Cubic Graph with 4 vertices and $3 \mathrm{deg}$.

\section{Isomorphism Graph}

Isomorphism in the graph can be defined by two graphs $G$ and $H$ that apply a bijective function between the vertex sets of $G$ and $H$,

$$
f: v(G) \rightarrow v(H)
$$

such that any two vertices $v$ and $u$ of $G$ are adjacent in $G$ if and only if $f(u)$ and $f(v)$ are adjacent in $H$ [8]. The illustration of two graphs which are isomorphic is described in Fig. 4.
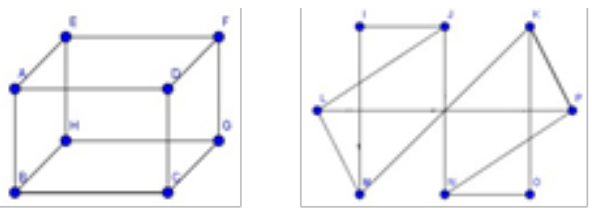

Fig. 4. Two graphs which are isomorphic.

Isomorphism representation can also be seen from its matrix adjacency. If the adjacency matrices are the same meaning the graph is isomorphic.

\section{E. Automorphism Graph}

Automorphism of the graph is graph $G$ isomorphism mapped onto itself. Automorphism of graph $G=(V, E)$ is a permutation of $\sigma$ from vertices set $V$ form pair of vertices $(u, v)$ form an edge if and only if the pair $(\sigma(u), \sigma(v))$ also form an edge [9].

\section{F. Symmetric Graph}

A graph $G$ is symmetric (symmetric graph) if given any two pairs of adjacent vertices $u_{1}-v_{1}$ and $u_{2}-v_{2}$ of $G$, there is an automorphism $f: V(G) \rightarrow V(G)$ such that $f\left(u_{1}\right)=u_{2}$ and $f\left(v_{1}\right)=v_{2}[10]$.

In other words, a graph is symmetric if its automorphism applied in that graph.

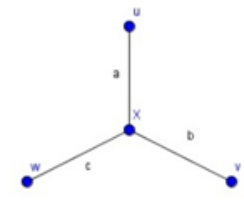

Fig. 5. Symmetric graph.

Symmetric properties of the graph in Fig. 5 can be verified by doing permutation on the vertex and edge.
TABLE I

PERMUtATION OF VERTEX AND EDGE IN Fig. 5.

\begin{tabular}{|l|l|l|}
\hline Symmetric & Vertex Permutation & Edge Permutation \\
\hline Identity & $(u)(v)(w)(x)$ & $(a)(b)(c)$ \\
\hline $120^{\circ}$ rotation & $(x)(u v w)$ & $(a b c)$ \\
\hline $240^{\circ}$ rotation & $(x)(u v w)$ & $(a b c)$ \\
\hline edge a reflection & $(x)(u)(v w)$ & $(a)(b)(c)$ \\
\hline edge b reflection & $(x)(v)(u w)$ & $(b)(a c)$ \\
\hline edge c reflection & $(x)(w)(u v)$ & $(c)(a b)$ \\
\hline
\end{tabular}

\section{G. Petersen Graph}

Petersen graph is a graph that has 10 vertices and 15 edges. This graph was constructed in 1989 by Julius Petersen. Petersen graph is generally drawn as a pentagon with a pentagram inside. But there are also other forms of Petersen graph as illustrated on Fig. 6. Petersen graph is an ideal example of symmetric cubic graph.

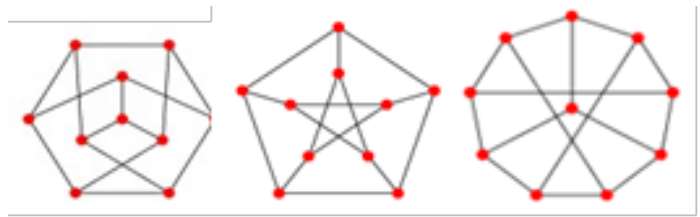

Fig. 6. Three forms of Petersen graph.

\section{H. Tracks and Circuits}

Tracks are the length from the initial node $v_{0}$ to the destination node $v_{n}$ with the total node is $n$. The track length is the number of sides in that track. Meanwhile, the circuit is a track that is started at $v_{0}$ and ended at the same $v_{0}$ [?].

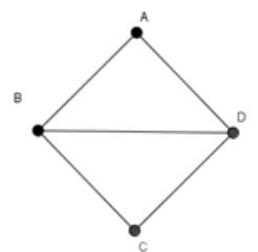

Fig. 7. Tracks and circuits of graph G.

In Fig. 7, the tracks of graph $G$ are A, B, C, D with a row of side $(A, B),(B, C),(C, D),(D, A)$. While the circuits of that graph are $A, B, C, D, A$..

\section{Euler Graph}

A graph $G$ is an euler graph if there is euler circuit in that graph. If there is only euler track in that graph, then the graph is called Semiuler Graph [4].

Euler tracks of graph in the left are: A, F, E, D, C, E, G, C, B, G, G, F, F, A. While, the graph on the right does not have any Euler circuit. 


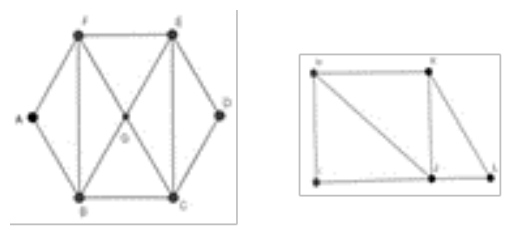

Fig. 8. The left part is an Euler graph, whereas the right one is not an Euler graph.

\section{J. Hamilton Graph}

A graph $G$ is said to be Hamiltonian or Hamilton Graph when the graph contains a Hamilton circuit which passes through each vertex in the graph exactly once. Further if the graph only has Hamilton track then it is called Semi-Hamilton Graph.

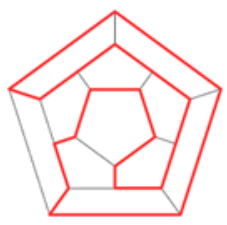

Fig. 9. Hamilton track on the graph.

\section{K. Girth}

Girth on the graph is defined as the length of a shortest shekel contained in a graph. If the graph does not have any shekel, then it is said to have infinite girth (infinity) [3].

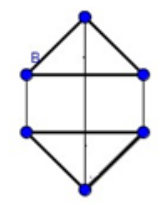

Fig. 10. Cubic Graph with 3 girths.

\section{Hipohamilton Graph}

Graf Hipohamilton is a graph $(G)$ in which if a vertex on that graph is omitted, the graph will be Hamilton Graph. Petersen graph can be classified as this type of graph.
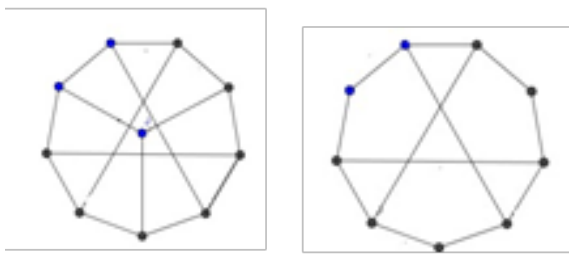

Fig. 11. Petersen Graph transformed to Hipohamilton graph.

\section{Graph Connectivity}

In a cubic graph genetic process, supporting partitions are needed to merge between graphs. A graph can be transformed, so the necessary parts are derived. A bond of relationship between vertex and edge is called connectivity (connectivity). Graph connectivity can be classified into two: the connectedness of vertex (vertex connectivity) and the connectedness of edge (edge connectivity). Connectivity in the graph means that between vertex and edge in the graph has a bond that if the connectivity is omitted, then a graph be unconnected (disconnect). Part that can be omitted in the connectivity (connectivity) is called cutset.

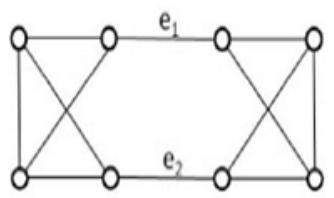

Fig. 12. The connectivity of edge and cutset.

\section{N. Cutset}

In Fig. 12, $e_{1}$ and $e_{2}$ are cutset edge. In the graph, there is edge connectivity because edge $e_{1}$ and $e_{2}$ connect two parts of the graph to be a whole graph. This principle will be used to construct genetic partitions on a further cubic graph.

\section{O. $\mathrm{N}$-Cracker}

$\mathrm{N}$-cracker defined as n-edge that is not adjacent (adjacent) which if it is removed would make the graph is not connected. $N$ denotes the number of adjacent edge. If an edge which is predicted to be n-cracker is omitted, but in fact it does not make a graph is not connected, then still it is named as common cutset. $N$-cracker only applies on Cubic Graph [5].

\section{P. Gene}

Cubic Graph has a maximum degree of 3. Thus, automatically its $n$-cracker is $n<3$, with $n \in\{1,2,3\}$. An $n$-cracker with $n \in\{1,2,3\}$ is called cubic cracker. Moreover, if a Cubic Graph does not have any cubic cracker then that graph is called gene [5].

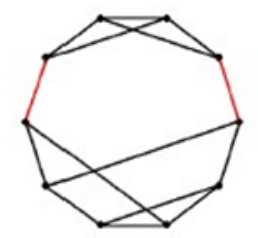

Fig. 13. Cubic graph with 2 crackers.

\section{Q. Parthenogenesis}

The next genetics process of cubic graph is parthenogenesis. Similar to breeding, parthenogenesis process in cubic graph can be divided into three types [5]: 
1) Parthenogenesis Type I: This happens on cubic graph with 1-cracker, by inserting diamond graph into cubic graph by cutting the segment of graph's cutset. Type I of parthenogenesis will produce a single graph with a diamond graph on the edge connectivity.

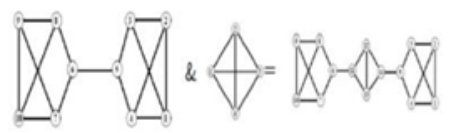

Fig. 14. Parthenogenesis type I.

2) Parthenogenesis Type II: This happens on cubic graph with 2-cracker, by inserting bridge graph (bridge graph) into edge connectivity of cubic graph. Type II of parthenogenesis will produce a single graph with a bridge graph on the edge connectivity.

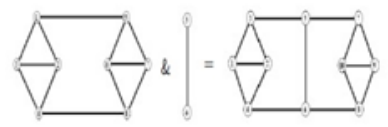

Fig. 15. Parthenogenesis type II.

3) Parthenogenesis Type III: This happens on cubic graph with 3-cracker, by inserting a triangle gaph (triangle graph) into a cubic graph. Type III of parthenogenesis will produce a single cubic graph with a triangle graph on the edge connectivity.

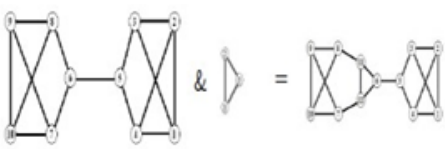

Fig. 16. Parthenogenesis type III.

\section{Results And Discussions}

Some steps on the process of parthenogenesis:

1) Identify the nature and character of Petersen Graph that will be studied. Based on the review of literature as elaborated previously, Petersen Graph has some characteristics as follows:

a) Petersen Graph has 10 vertices and 15 edges.

b) Petersen Graph is a symmetric Cubic graph.

c) Petersen Graph is Hamiltonian.

d) Petersen Graph has grith.

e) Isomorphism with another form of Petersen graph.

2) Deciding parthenogenesis type used (cf. Fig. 17) Based on the figure illustration, Petersen Graph is 3 crackers in the center of the graph, thus, parthenogenesis process used is type III.

3) Some steps in parthenogenesis process are as follows:

a) Segment cutting of Petersen graph (cf. Fig. 18)

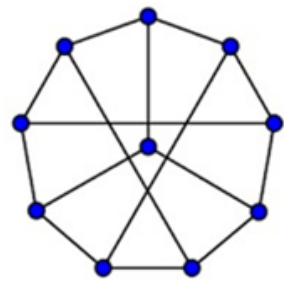

Fig. 17. A graph.

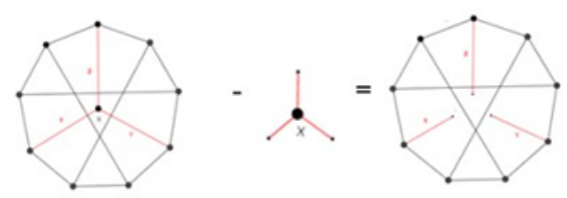

Fig. 18. Some graphs.

b) Merging with gene (cf. Fig. 19)

c) Deciding the graph as the result of parthenogenesis (cf. Fig. 20)

4) Study on the new graph as resulted from parthenogenesis

a) There is an alteration on the vertex and the edge (cf. Fig. 21).

b) Isomorphism is applied (cf. Fig. 22)

c) The reduction on the total of girth (cf. Fig. 23)

d) There is no change on the nature of the Hamiltonian (cf. Fig. 24)

e) In form of Symmetric Graph (cf. Fig. 25)

Permutation table of the new graph as resulted from parthenogenesis listed below:

TABLE II

PERMUTATION TABLE OF THE NEW GRAPH

\begin{tabular}{|l|l|l|}
\hline Symmetric & Vertex permutation & Edge permutation \\
\hline Identity & $(K)(L)(M)(N)$ & $(a)(b)(c)(d)(e)$ \\
& $\begin{array}{l}(O)(P)(Q)(R) \\
(S)(T)(U)(V)\end{array}$ & $\begin{array}{l}(f)(g)(h)(i)(j) \\
(k)(l)(m)(n) \\
(o)(p)(q)(r)\end{array}$ \\
\hline $\begin{array}{l}\text { Reflection on } \\
\text { the edge } o\end{array}$ & $(K)(T)(L S)$ & $(o)(k)(n)(r)$ \\
& $(M R)(U R)$ & $(a b)(c d)(g h)$ \\
$(N Q)(O P)$ & $(I j)(e m)(e f)(p q)$ \\
\hline
\end{tabular}

\section{CONCLUSIONS}

Based on the discussion above, it can be concluded that:

1) The process of parthenogenesis in Petersen graph elaborated parthenogenesis type III.

2) The process of parthenogenesis in Petersen graph was begun with the omission of the segment; in this case, there were three edges which are the cutset edge.

3) The nature and the characteristics generated in the new graph (Y) as resulted of parthenogenesis type III in Petersen graph are as follows:

4) There was an increment of vertex and edge. Started from 10 vertices to 12 vertices and started from 15 edges to 18 edges. 


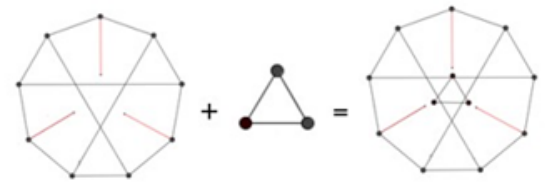

Fig. 19. Some graphs.

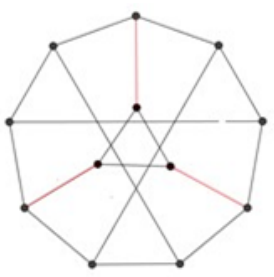

Fig. 20. A graph.

5) Parthenogenesis in Petersen graph did not change the nature of isomorphism between Petersen which also experienced the process of parthenogenesis.

6) Parthenogenesis changed girth of Petersen graph. Petersen graph has 5 girth, after parthenogenesis process, girth became 3 .

7) There was no change on the Hamiltonian nature of the graph. Included in that group. As referred to the permutation table of the new graph.

8) New graph $(Y)$ as resulted from Petersen graph parthenogenesis process has some similarities to Petersen graph; that is symmetric graph.

\section{REFERENCES}

[1] G. Nirmala and D. Ramprasad, "Application of genetic algorithm in graph theory," International Journal of Scientific and Research Publications, vol. 2, no. 7, 2012.

[2] Nurhayati, "Sejarah teori graph," 2012.

[3] R. Diestel, Graph Theory. Springer Verlag, 2000.

[4] F. Harary and E. Palmer, "Graphical enumeration," Academic Press, New York, UBKA math, vol. 9, p. 74, 1973.

[5] P. Baniasadi, V. Ejov, J. Filar, and M. Haythorpe, Genetic Theory for Cubic Graphs. Springer International Publishing, 2016, pp. 1-26.

[6] R. Trudeau, Introduction to graph theory. Courier Corporation, 2013.

[7] F. Harary, "Graph theory," 1969.

[8] N. Biggs, Algebraic Graph Theory. Cambridge University Press, 1993.

[9] R. Frucht, "Graphs of degree three with a given abstract group," Canadian J. Math, vol. 1, pp. 365-378, 1949.

[10] C. Godsil and G. Royle, "Algebraic graph theory, volume 207 of graduate texts in mathematics," 2001.
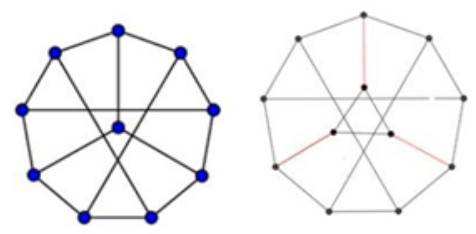

Fig. 21. Some graphs.

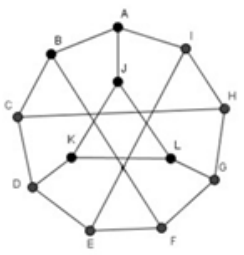

(a)

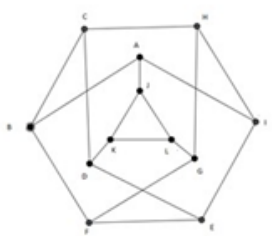

(b)

Fig. 22. Several graphs.
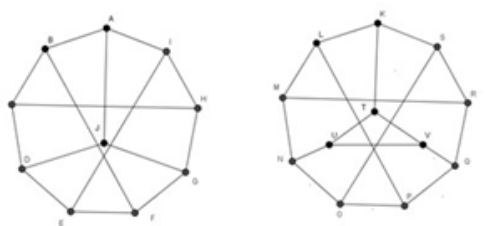

Fig. 23. Two graphs.
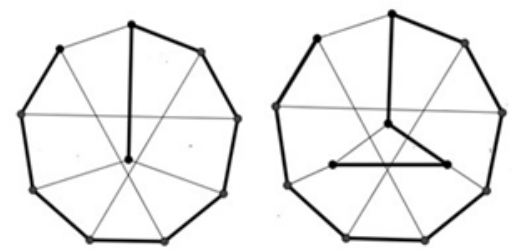

Fig. 24. Two graphs.

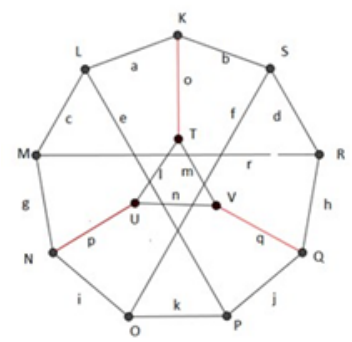

Fig. 25. A graph. 